\title{
Expression of Hemagglutinin-Neuraminidase (HN) and Fusion (F) Epitopes of Newcastle Disease Virus (NDV) in Chlamydomonas reinhardtii
}

\author{
Amir Ghaffar Shahriari ${ }^{1, *}$, Alireza Afsharifar ${ }^{2}$, Maziar Habibi-Pirkoohi ${ }^{3}$ \\ ${ }^{1}$ Department of Agriculture and Natural Resources, Higher Education Center of Eghlid, Eghlid, Iran \\ ${ }^{2}$ Plant Virology Research Centre, College of Agriculture, Shiraz University, Shiraz, Iran \\ ${ }^{3}$ Research and technology institute of plant production, Shahid Bahonar University of Kerman, Iran
}

*Corresponding author: Shahriari.ag@eghlid.ac.ir

\begin{abstract}
Owing to their unique characteristics which combines the properties of both prokaryotes and eukaryotes, microalgae have emerged as an ideal platform for heterologous production of recombinant proteins including subunit vaccines. In an attempt to develop recombinant vaccine against Newcastle Disease, an agrobacterium-mediated genetic transformation was carried out to express a chimeric gene construct including Hemagglutinin-Neuraminidase (HN) and Fusion (F) epitopes of Newcastle Disease Virus (NDV) in Chlamydomonas reinhardtii. Four tandem repeat of HN epitope with $96 \mathrm{bp}$ length followed by three tandem repeat of F epitope of NDV with 153bp length were used. Microalgal cells (Chlamydomonas reinhardtii) were co-cultivated with Agrobacterium tumefaciens cells harboring foreign gene construct and then transferred to selection medium. Single colonies representing putative transformation events were screened in selection medium enriched with kanamycin. PCR assay confirmed integration of F-HN sequence in microalgal nuclei. RT-PCR assay showed that the F-HN sequence was expressed in transformed colonies. Finally, translation of the foreign gene was confirmed by protein dot blotting, western blot and Elisa assay. The results of this experiment may contain both research and practical implications.
\end{abstract}

Keywords: Chlamydomonas reinhardtii; Newcastle Disease; recombinant vaccine; transformation.

Abbreviations: F_Fusion; HN_Hemagglutinin-Neuraminidase HN; NDV_Newcastle Disease Virus; PCR_Polymerase chain reaction.

\section{Introduction}

Green plants are usually considered as ideal platforms for production of oral recombinant vaccine owing to various advantages including cost-effective production process, feasibility for using as raw products, presence of cell wall that protects the antigen against gastric digestion, possession of eukaryotic posttranslational modification machinery, as well as others (Habibi-Pirkoohi and Mohkami, 2015). Despite numerous advantages of green plants over other heterologous expression systems such as bacteria, yeast and insect cell culture, low expression level of the antigen in plant hosts is still a main challenge for commercialization of plant-based recombinant vaccines. In this regard, applying consecutive epitopes represents an effective way to boost immunogenicity of the recombinant antigens (Shahriari et al., 2016).

During recent years, green microalgae have emerged as highly suitable production platforms for production of various recombinant vaccines. Application of microalgae as a novel system for production of recombinant vaccines is a potentially valuable option considering its significant advantages over microbial and plant systems (Specht et al., 2010). Microalgae can be grown in fairly inexpensive media; they growth fast like prokaryotes and reach maximum biomass in short time and, at the same time, they have eukaryotic translation machinery which allows for correct folding of the foreign antigen. Overall, microalgae possess advantages of both eukaryotic and prokaryotic species usually used for recombinant protein expression (Gimpel et al., 2015). Furthermore, microalgae are generally recognized as safe (GRAS), and thus can be eaten fresh which represents a major property for development of oral recombinant vaccines (Habibi-Pirkoohi et al., 2014). Since the prominent work of Kumar et al (2004), agrobacteriummediated transformation of the microalgae has emerged as a fairly quick and easy tool compared to conventional methods such as glass beads, protoplast transformation and so on.

Newcastle Disease Virus (NDV) is the causative agent of Newcastle disease which represents a major challenge for poultry industry. Fusion (F) and Hemagglutinin-Neuraminidase (HN) are glycoproteins that are critical for virulence and can stimulate immune system. Amino acids $65-81$ of $\mathrm{F}$ protein and 346-353 of $\mathrm{HN}$ have been identified as the most important 
immunogenic sites for induction of antibody (Shahriari et al., 2016). Considering importance of the disease and some problems of conventional vaccination, many researchers have attempted to develop efficient plant-based recombinant vaccine against NDV (Ge et al., 2014; Lai et al., 2013; Ngu et al., 2018). The main progress in development of anti-NDV recombinant vaccine was achieved by expression of the virus epitopes in tobacco cell culture. This is the only licensed plantproduced vaccine against ND (Rybicki, 2009). However, tobacco is an alkaloid-rich plant that can't be used orally and thus requires further purification steps which intensifies production costs (Yusibov et al., 2015). In contrast, microalgae can be used freshly as they are GRAS organisms; the property that makes them suitable platform for scalable recombinant vaccine production (Habibi-Pirkoohi et al., 2014). Based on these consideration, the present research was conducted to express consecutive sequences of $\mathrm{F}$ and $\mathrm{HN}$ epitopes in the green microalga Chlamydomonas reinhardtii via agrobacterium-mediated genetic transformation.

\section{Results}

At the end of co-cultivation period, some colonies of $C$. reinhardtii appeared in the selection medium which were harvested and used for PCR analysis (Fig. 1).

\section{Primary screening of the colonies}

As is the case with all microalgae transformation procedures, the first indicator of algal cells is presence of single colonies in the medium which differs with an even lawn often observed in normal culture. Emergence of single colonies in the selection medium implied that the untransformed cells were destroyed due to presence of selection agent (kanamycin) and only colonies harboring the transgene were able to survive and growth. Since the construct was not equipped with reporter genes, no other phonotypical measure could be used for identification of the transformed colonies. Occurrence of genetic transformation was further verified through conducting a PCR assay. In PCR, a band of $810 \mathrm{bp}$ was observed in the lane corresponding to genomic DNA of putative transformed colonies. The results of PCR analysis indicated that transgene was present in putative transformed colonies. No PCR band was observed in negative control; suggesting reliability of PCR verification assay (Fig. 2).

\section{Transgene expression at transcription level}

Since heterologous expression of F-HN protein in microalgae cells was the goal of the present study, transcription of this fusion protein was also analyzed in PCR-positive colonies. For this purpose, expression of $F$ and $H N$ epitopes was measured semi-quantitatively at transcription level by RT-PCR. As seen in figure 3, RT-PCR assay also confirmed integration of transgene in transformed colonies. Lanes 3-4 representing transformed colonies showed positive results (expected 307bp bands) with the sharpest band was observed in lane 5 . The bands observed for transformed colonies were in the same size of positive control; while no band was observed in the lane devoted to wild type sample.

\section{Evaluation of the recombinant protein}

Positive colonies of RT-PCR assay were further studied for verification of $\mathrm{F}-\mathrm{HN}$ expression at protein level. This was conducted by protein dot blot assay. As can be seen from figure 4, positive response was observed for the sample obtained from transgenic colony while a very faint dot was achieved for wild type cells. This finding imply that the F-HN fusion gene was successfully translated to the corresponding recombinant protein in microalgae cells.

Although dot blot assay represents a robust verification of translation of recombinant proteins in host cells, it is not a suitable method for quantitative evaluation of transgene expression. Therefore, ELISA assay was also performed for quantification of heterologous expression of $\mathrm{F}-\mathrm{HN}$ protein. ELISA results (Fig. 5) confirmed that the recombinant protein was expressed fairly high is the transformed colonies. Although the detection signal in positive control was apparently higher than that of transformed colonies, the results clearly showed that interested recombinant protein was in large quantity in the transformed colonies as can be inferred by comparing transformed colonies and negative control $(p<0.05)$.

At the final part of the experiment, western blotting was conducted to visualize the recombinant protein at the membrane. The result of western blotting (fig. 6) indicated the expected protein band in the sample obtained from transformed colony; whereas no band was observed for the sample gained from wild type colonies. Confirming the results of previous assays, the output of western blotting assay showed that the recombinant protein was successfully expressed in the transformed colonies.

\section{Discussion}

In this research, utility of microalgae as a potentially inexpensive and affordable platform for production of recombinant vaccine has been investigated. Microalgae have been regarded as a scalable choice for production of therapeutic substances. Gong et al (2011), for example, reviewed technical progress in production of recombinant proteins in microalgae and concluded that these unicellular microorganism have various advantages that make them suitable platform as bioreactors for large scale production of therapeutics. Potvin and Zhang (2010) also remarked high potential of microalgae for production of various compounds of high pharmaceutical value. The results obtained in the present study reconfirm suitability of the fresh water microalga $C$. reinhardtii as green cell factory with high potential for molecular farming. This is in line with previous reports implying that this unicellular microalga possesses considerable potential for commercial production of various types of pharmaceutics. Rasala and Mayfield (2015) reported that $C$. reinhardtii is a suitable platform for production of industrial enzymes, hormones, antibodies and recombinant vaccines. Similarly, Gong et al. (2011) argued that C. reinhardtii 


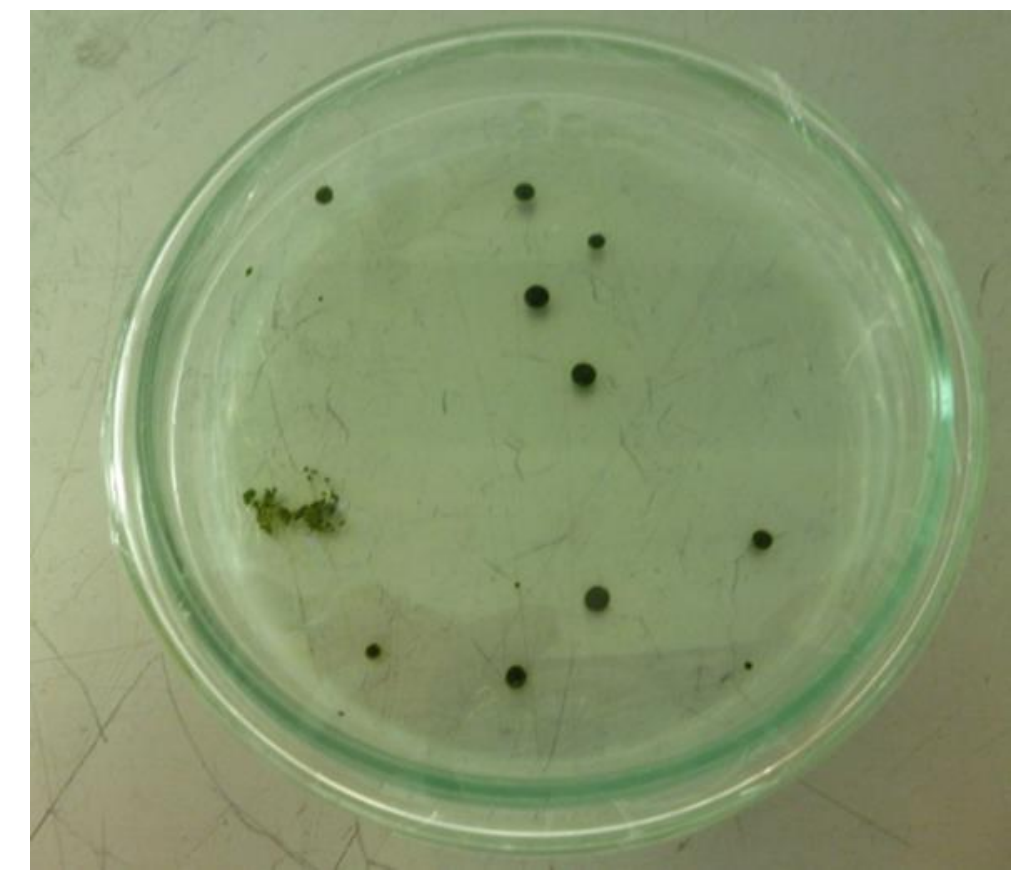

Fig 1. Emergence of putative transformed colonies in selection medium.

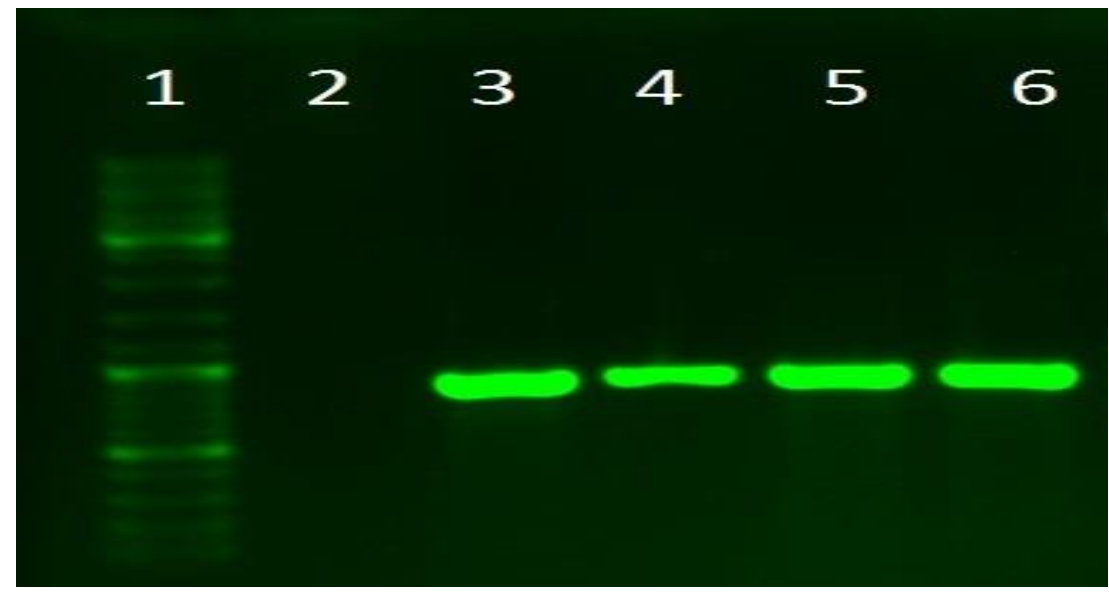

Fig 2. PCR detection of recombinant gene in transformed colonies; 1 : ladder, 2: wild type DNA sample, 3-5: transformed colonies; 6: plasmid pBI121-NDV.

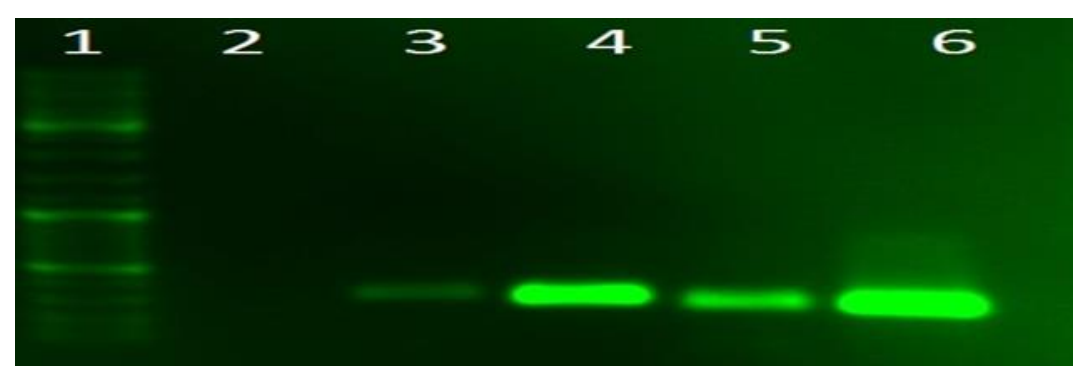

Fig 3. RT-PCR results for evaluation of transgene transcription in transformed colonies. 1: size marker, 2: wild type DNA sample, 3-5: transformed colonies; 6: plasmid pBI121-NDV. 


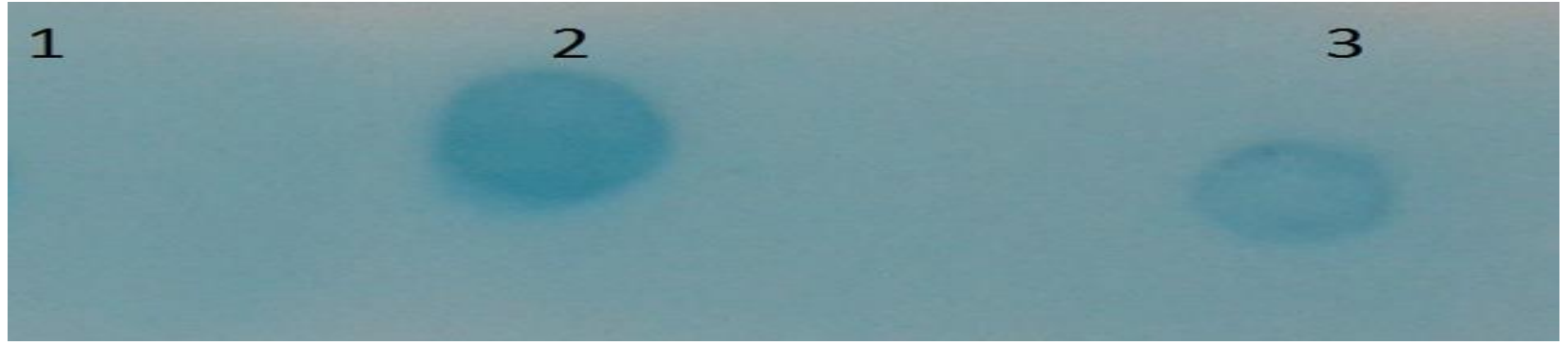

Fig 4. Dot blot analysis results. 1: wild type; 2-3: transformed colony.

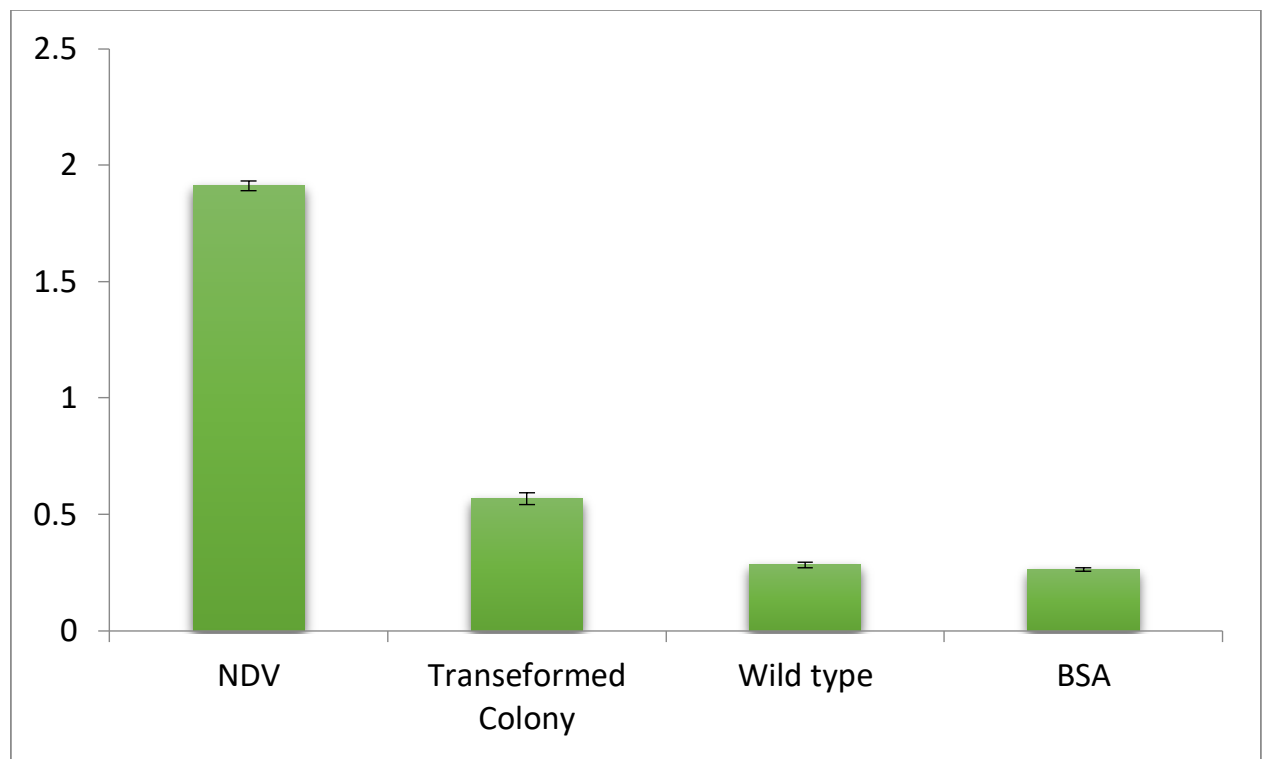

Fig 5. ELISA results for quantification of recombinant proteins. The sharpest signal was observed in positive control followed by transformed colony $(p<0.05)$.

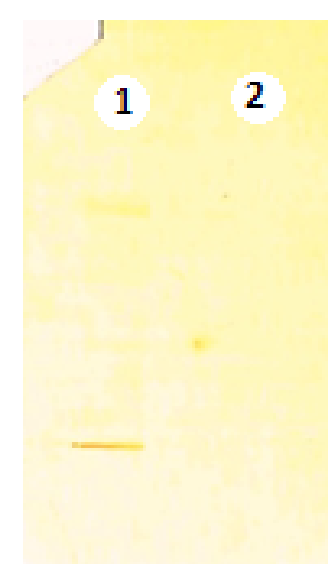

Fig 6. Western blot analysis of transgenic colonies. Lane1: protein sample of transgenic colony, lane 2: protein sample of wild type colony 


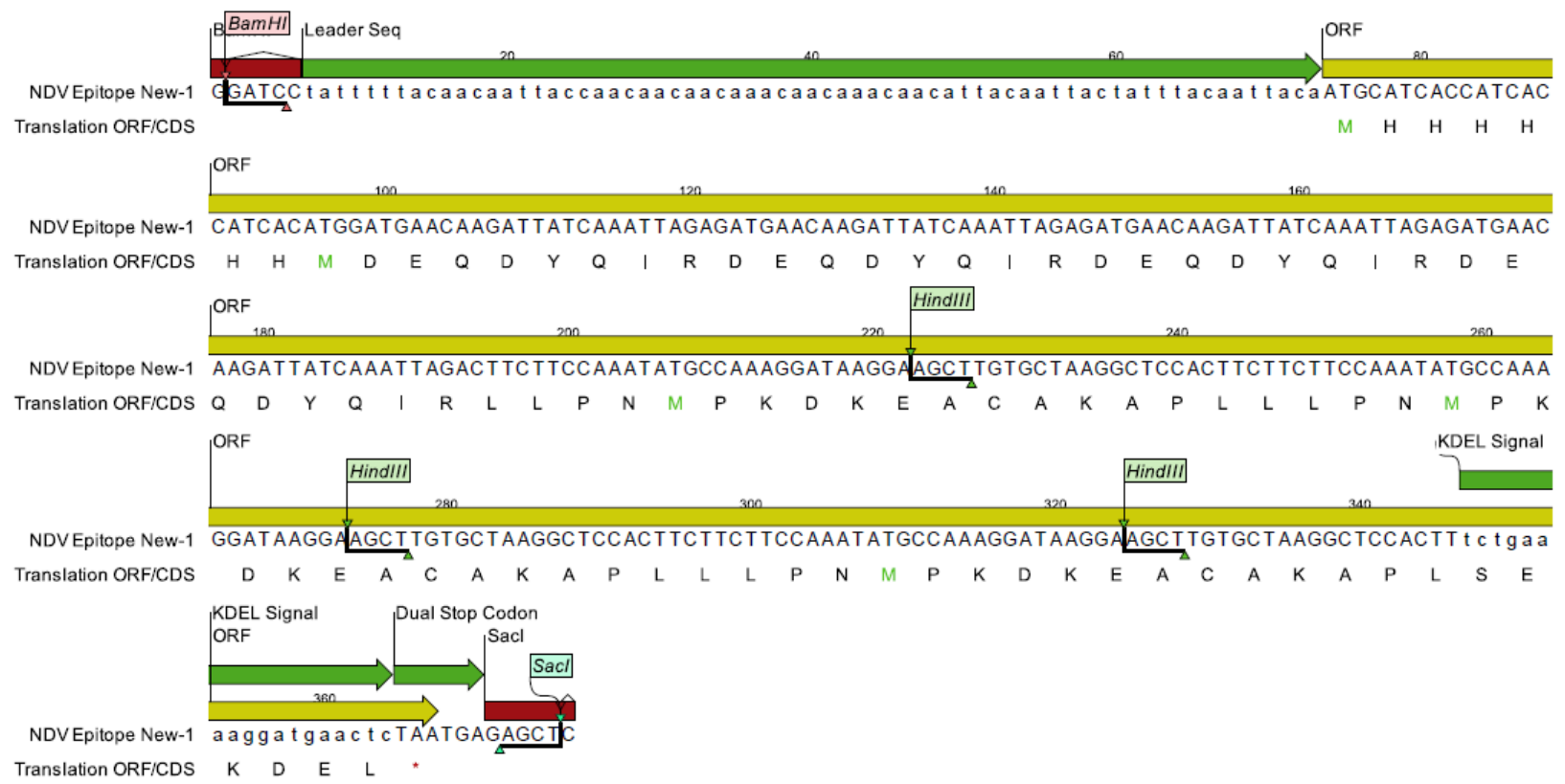

Fig 7. Schematic presentation of the gene construct. BamHI restriction site and AUG initiation codon were inserted at beginning of coding sequence; SEKDEL

can be used for production of different pharmaceutics at commercial level. Reviewing recombinant vaccine production in green plants, Habibi-Pirkoohi and Mohkami (2015) pointed out that microalgae such as $C$. reinhardtii can be regarded as ideal platform for production of recombinant subunit vaccines (Habibi-Pirkoohi and Mohkami, 2015). Other species of microalgae have also been used for production of recombinant pharmaceutical materials (Salbitani et al., 2014).

As approved in this research, the transgene was correctly translated and folded within microalgal cells; suggesting that microalgae is a reliable host for biomanufacturing of recombinant vaccines. This finding agrees with those reported by other researchers studying production of recombinant vaccines in microalgae systems. In a comprehensive descriptive paper, Specht et al (2010) noticed that microalgae can be a safe, inexpensive and scalable platform for production of recombinant vaccines. Sun et al (2013) successfully expressed fusion protein containing the VP1 gene and the cholera toxin B subunit in C. reinhardtii. Syndrome virus protein 28 (VP28) was also expressed in the cells of the same species (Surzycki et al., 2009). More recently, Habibi-Pirkoohi et al (2014) tried to express Food and Mouth Disease Virus (FMDV) VP1 antigen in C. reinhardtii. The encoding gene was successfully integrated in the host genome; however, the transformed colonies were not stable and lost a couple of days after transformation (HabibiPirkoohi et al., 2014). In contrast, in the present study, the transgene was integrated in $C$. reinhardtii nuclei and the colonies were able to growth.

Overall, we used C. reinhardtii as a platform for production of anti-NDV recombinant vaccine. Regarding its numerous advantages over conventional systems such as yeast, bacteria and even higher plants, microalgal system may be of great importance for large scale production of subunit vaccine against NDV. However, we did not investigate immunogenicity of the recombinant protein in this research and hence, we can't claim development of a new system for production of anti-NDV vaccines. Further research in needed to approve usability of this system as cell factory for scalable production of subunit vaccine against ND. At least, our experiment paves the road for application of $C$. reinhardtii as a potent system to produce anti-NDV vaccines at large scale. Investigation of immunogenicity of recombinant $\mathrm{F}-\mathrm{HN}$ peptide may be the subject of future researches.

\section{Materials and Methods}

\section{Design of gene construct}

Four tandem repeats of HN epitope followed by three tandem repeats of $F$ epitope were used as the core part of gene construct. The initiation codon (AUG) followed by histidine tag (18 bp) and omega sequence as ribosome binding (67 bp) site were attached in upstream of the gene. The endoplasmic reticulum signal (SEKDEL) was added to $3^{\prime}$ end just before termination codon. Finally, two restriction sites namely BamHI and $\mathrm{SaCl}$ were added to $5^{\prime}$ and $\mathrm{3}^{\prime}$ ends. The final length of the gene was $376 \mathrm{bp}$. Gene construct was designed by CLC software (Fig. 7). The resulting gene construct was synthesized and cloned in pGH vector by Gene ray Company. Construction of binary vector have been described elsewhere (Shahriari et al., 2016).

\section{Microalgae transformation}

A sterile and fresh culture of Chlamydomonas reinhardtii was obtained from Research and Technology Institute of Plant 
Production, Shahid Bahonar University, Iran. C. reinhardtii cells were transformed using the method reported by kumar et al (2004) with some modifications. Briefly, $150 \mu$ l of microalgal suspension in log phase was transferred to solid Tris Acetate Phosphate (TAP) medium and incubated in light for 2 days to form algal lawn. A fresh Agrobacterium culture $(A 600=0.5)$ grown in liquid LB medium containing appropriate antibiotics (20 mg/l rifampicin and $50 \mathrm{mg} / \mathrm{l} \mathrm{kanamycin)} \mathrm{was} \mathrm{centrifuged} \mathrm{in}$ $6000 \mathrm{~g}$ for $5 \mathrm{~min}$, the supernatant was discarded and the bacterial pellet was resuspended in $200 \mu \mathrm{l}$ TAP broth. The bacterial suspension was spread to the thin layer of Chlamydomonas lawn growing on agar plate. The cocultivation plates were incubated for 2 days at $25^{\circ} \mathrm{C}$. The cells were then harvested and washed twice with liquid TAP medium containing $500 \mathrm{mg} / \mathrm{l}$ cefotaxime to eliminate bacterial residues. The washed Chlamydomonas cells were cultured in solid selection medium containing $50 \mathrm{mg} / \mathrm{l}$ Kanamycin and 500 $\mathrm{mg} / \mathrm{l}$ cefotaxime.

\section{Detection of transformed colonies}

Appearance of single colonies in selection media was taken as the first indicator of transformation event. Since microalgae cells are naturally sensitive to Kanamycin, only transformed colonies harboring resistance gene could growth in selection media. However, since this is not a reliable measure to evaluate transformation, PCR test was performed. Half of each transformed colony was screened for presence of the gene of interest using specific primers with sequences of $5^{\prime}$ TCATTGCGATAAAGGAAAGGC- 3' and

5' AATGTATAATTGCGGGACTC 3'. Cells were resuspended in Tris-EDTA solution and heated to $95^{\circ} \mathrm{C}$ for 10 minutes. The cell lysate was used as a template for PCR reactions. The other halves of the colonies with positive PCR results were grown in liquid selection medium for further analysis. DNAs of wild type colonies and plasmid pBI121-NDV were used as negative and positive control; respectively. PCR product was segregated on $1 \%$ agarose gel.

\section{RT-PCR}

Expression of the transgene was evaluated at transcription level by reverse transcription polymerase chain reaction (RTPCR) assay. Total RNA was extracted from fresh colonies according to DNA-free RNA isolation method. Complementary DNA (cDNA) was synthesized via reverse transcription using oligo(dT)20 primer. The resulting cDNA mixtures were used as templates for RT-PCR. cDNA of wild type colonies and pF-HN epitopes plasmid were used as negative and positive controls; respectively.

\section{Detection of the recombinant protein}

Protein was extracted from the colonies using conventional Tris- $\mathrm{HCl}$ method. For dot blotting assay, $5 \mu \mathrm{l}$ of the protein extracted from transformed and wild type colonies was dotted on nitrocellulose membrane and the membrane was left to dry at $37^{\circ} \mathrm{C}$. Unwanted antibody reactions were blocked by incubating the membrane with Bovine Serum Albumin (BSA).
The membrane was then incubated with anti-His-tag conjugated with $\mathrm{Hrp}$ at $37^{\circ} \mathrm{C}$. The membrane was washed three times with PBS/PBST and then incubated with TMB substrate.

Recombinant protein expression was quantified by ELISA. ELISA plate was coated with total soluble proteins from the wild type and the transformed plants and purified NDV particles (commercial vaccine) at $37^{\circ} \mathrm{C}$ for one hour, followed by incubation with $1 \%$ bovine serum albumin (BSA) in PBS for 2 $\mathrm{h}$ at $37{ }^{\circ} \mathrm{C}$ to prevent non-specific binding. The well was washed by PBST/PBS and incubated with anti-NDV serum. Afterwards, it was incubated with horseradish peroxidase conjugated with anti-rabbit IgG (1:1500). The anti-NDV serum was prepared after three times of immunization in rabbit with the inactivated V4 NDV particles and diluted at 1:1000 in PBST. Wells were developed with TMB substrate. The color reaction was stopped by $2 \mathrm{~N} \mathrm{H} 2 \mathrm{SO} 4$ and read at $450 \mathrm{~nm}$ of wavelength.

For western blot analysis, Protein samples were resolved by $15 \%$ SDS-polyacrylamide gel electrophoresis and transferred onto nitrocellulose membrane at $130 \mathrm{~mA}$ for $2 \mathrm{~h}$. To prevent nonspecific antibody reactions, the membrane was blocked with BSA. After blocking with BSA, proteins were allowed to react with specific antibodies and the membrane was then washed using PBST/PBS, and incubated with an alkaline phosphatase labeled anti-mouse anti-rabbit IgG antiserum for $1 \mathrm{~h}$ at $37 \stackrel{\circ}{ } \mathrm{C}$. After washing three times, the reaction was developed by addition of the substrate.

\section{Conclusion}

The present research was conducted to evaluate appropriateness of microalgae as a fairly inexpensive and easyto-use platform for development of subunit vaccines. This research contribute to current literature in the field of both applied phycology and recombinant DNA technology by providing evidence denoting applicability of genetic transformation tools for production of subunit vaccines. Moreover, our results again confirmed suitability of microalgae as platform for production of recombinant therapeutic proteins. Microalgae have tick cell walls protecting the expressed antigen from digestion by hydrolyzing enzymes in stomach medium, they can be grown without so much expense and their biomass accumulation can be achieved in a short time. Moreover, microalgae such as $C$. reinhardtii are generally recognized as safe (GRAS), so they can be eaten fresh and this enhances the immunogenic potential of the expressed vaccine within algal cell. In general, the results obtained in this research and similar studies show that recombinant vaccines can be expressed in and harvested from transgenic microalgae in a fairly inexpensive and easy way. Obviously, further studies are required for optimization of recombinant sub-unit vaccines production in $C$. reinhardtii. Future researches may pave the road for commercial production of vaccines in microalgae systems.

\section{Conflict of interest}

There is no conflict of interest. 


\section{References}

Ge J, Wang X, Tian M, Wen Z, Feng Q, Qi X, Bu Z (2014) Novel in-ovo chimeric recombinant newcastle disease vaccine protects against both newcastle disease and infectious bursal disease. Vaccine. 32: 1514-1521.

Gimpel J A, Hyun J, Schoepp N, G, Mayfield S (2015) Production of recombinant proteins in microalgae at pilot greenhouse scale. Biotechnol Bioeng. 112: 339-345.

Gong Y, Hu H, Gao Y, Xu X, Gao H (2011) Microalgae as platforms for production of recombinant proteins and valuable compounds: progress and prospects. J Ind Microbiol Biot. 38: 1879-1890.

Habibi-Pirkoohi M, Mohkami A (2015) Recombinant vaccine production in green plants: State of art. J Cell and Mol Res. 7: 59-67.

Habibi-Pirkoohi M, Malekzadeh-Shafaroudi S, Marashi H, Moshtaghi N, Nassiri M, Mohkami A, Zibaee S (2014) Expression of foot and mouth disease virus (FMDV) capsid protein VP1 in chlamydomonas reinhardtii as a possible source of recombinant vaccine. Int J Plant Animal Env Scie. 4: 644-648.

Kumar SV, Misquitta RW, Reddy V, Rao B, Rajam MV (2004) Genetic transformation of the green alga chlamydomonas reinhardtii by Agrobacterium tumefaciens. Plant Sci.166: 731-738.

Lai KS, Yusoff K, Mahmood M (2013) Functional ectodomain of the hemagglutinin-neuraminidase protein is expressed in transgenic tobacco cells as a candidate vaccine against newcastle disease virus. Plant Cell Tiss Org. 112: 117-121.

Ngu L, N, Nji N, N, Ambada G, Ngoh AA, Priso N, Ghislain D, Tchouangueu TF (2018) Dendritic cell targeted HIV-1 gag protein vaccine provides help to a recombinant newcastle disease virus vectored vaccine including mobilization of protective CD8+ T cells. Immun Inflamm Dis. 6: 163-175.

Potvin G, Zhang Z (2010) Strategies for high-level recombinant protein expression in transgenic microalgae: a review. Biotechnol Adv. 28: 910-918.

Rasala BA, Mayfield S (2015) Photosynthetic biomanufacturing in green algae; production of recombinant proteins for industrial nutritional and medical uses. Photosynth Res. 123: 227-239.

Rybicki EP (2009) Plant-produced vaccines: promise and reality. Drug Discov Today. 14: 16-24

Salbitani G, Wirtz M, Hell R, Carfagna S (2014) Affinity purification of O-acetylserine (thiol) lyase from chlorella sorokiniana by recombinant proteins from Arabidopsis thaliana. Metabol. 4: 629-639.
Shahriari A, Bagheri A, Bassami M, R, Malekzadeh-Shafaroudi S, Afsharifar A, \& Niazi A (2016) Expression of hemagglutininneuraminidase and fusion epitopes of newcastle disease virus in transgenic tobacco. Electron J of Biotechn. 19(4): 3843.

Specht E, Miyake-Stoner S, \& Mayfield S (2010) Micro-algae come of age as a platform for recombinant protein production. Biotechnol Lett. 32: 1373-1383

Sun M, Qian K, Su N, Chang H, Liu J, \& Shen G (2003) Foot-andmouth disease virus VP1 protein fused with cholera toxin B subunit expressed in Chlamydomonas reinhardtii chloroplast. Biotechnol Lett. 25: 1087-1092.

Surzycki R, Greenham K, Kitayama K, Dibal F, Wagner R, Rochaix JD, Surzycki S (2009) Factors effecting expression of vaccines in microalgae. Biologicals. 37 (3):133-138.

Yusibov V, Kushnir N, Streatfield S J (2015) Advances and challenges in the development and production of effective plant-based influenza vaccines. Exp Rev vaccines. 14(4): 519535. 